\title{
ON THE UNIVALENT FUNCTIONS STARLIKE WITH RESPECT TO A BOUNDARY POINT
}

\author{
PAVEL G. TODOROV
}

\begin{abstract}
For the examined functions, we have obtained a structure formula and estimates for $|f(z) /(1-z)|$ and $|\arg (f(z) /(1-z))|$, the moduli of the partial sums of the coefficient series and the moduli of the coefficients.
\end{abstract} tions:

Recently, Robertson [1] introduced the following two classes of univalent func-

DEFINITION 1. Let $G^{*}$ denote the class of functions $f(z)$ analytic in $D=$ $\{z|| z \mid<1\}$, normalized so that $f(0)=1, f(1)=\lim _{r \rightarrow 1} f(r)=0$, and such that for some real $\alpha, \operatorname{Re}\left[e^{i \alpha} f(z)\right]>0, z \in D$. In addition let $f(z)$ map $D$ univalently on a domain starlike with respect to $f(1)$. Let the constant function 1 also belong to the class $G^{*}$.

DEFINITION 2. Let $G$ denote the class of functions $f(z)$ analytic and nonvanishing in $D$, normalized so that $f(0)=1$ and such that

$$
\operatorname{Re}\left\{\frac{2 z f^{\prime}(z)}{f(z)}+\frac{1+z}{1-z}\right\}>0 \quad(z \in D) .
$$

Robertson [1] conjectured that the classes $G^{*}$ and $G$ coincide. Recently, Lyzzaik [2] proved this conjecture.

Now we shall continue the study of the class $G$.

THEOREM 1. The function $f(z)$ belongs to the class $G$ if and only if $f(z)$ can be written in the form

$$
f(z)=(1-z) \exp \left\{-\int_{-\pi}^{\pi} \ln \left(1-z e^{-i t}\right) d \mu(t)\right\} \quad(z \in D),
$$

for some probability measure $\mu$ defined on the interval $[-\pi, \pi]$.

PROOF. It follows from (1) and a classic result due to Herglotz that

$$
\frac{2 z f^{\prime}(z)}{f(z)}+\frac{1+z}{1-z}=\int_{-\pi}^{\pi} \frac{1+z e^{-i t}}{1-z e^{-i t}} d \mu(t)
$$

holds in $D$ for some probability measure $\mu(t)$. A simple integration now yields the desired structure formula $(2)$ for $f(z)$.

THEOREM 2. For a fixed $z \in D$, we have the relation

$$
\{w \mid w=(1-z) / f(z), f(z) \in G\}=\{w|| w-1|\leqq| z \mid\}
$$

Received by the editors January 11, 1985 and, in revised form, May 10, 1985.

1980 Mathematics Subject Classification. Primary 30C45.

Key words and phrases. Univalent functions. 
where for $z \neq 0$ the equality holds only for the functions

$$
f(z)=\frac{1-z}{1-e^{i \omega} z}=1+\sum_{n=1}^{\infty}\left[e^{i n \omega}-e^{i(n-1) \omega}\right] z^{n} \in G, \quad \omega \in[-\pi, \pi] .
$$

ProOF. From (2) it follows that

$$
\ln \frac{1-z}{f(z)}=\int_{-\pi}^{\pi} \ln \left(1-z e^{-i t}\right) d \mu(t)
$$

holds in $D$. According to the Carathéodory principle $[3]$ (see also $[4$, p. 543; 5]), from (5) it follows that for a fixed $z \in D$ we shall have the relation

$$
\left\{\varsigma \mid \zeta=\ln \frac{1-z}{f(z)}, f(z) \in G\right\}=\mathrm{CH}\left\{\zeta \mid \zeta=\ln \left(1-z e^{-i t}\right), t \in[-\pi, \pi]\right\},
$$

where $\mathrm{CH}$ denotes the convex hull of the set in the braces. The function

$$
\varsigma=\ln w
$$

maps the $w$-plane out along the negative real axis onto the strip $\{\zeta \mid-\pi<\operatorname{Im} \zeta<\pi\}$. It is clear geometrically that the function (7) maps the disc $\{w|| w-1|\leqq| z|| z \mid,<1\}$ onto some convex domain lying in this strip. Therefore, for a fixed $z \in D$ from (6) the relation

$$
\left\{w \mid w=\frac{1-z}{f(z)}, f(z) \in G\right\}=\mathrm{CH}\left\{w \mid w=1-z e^{-i t}, t \in[-\pi, \pi]\right\}
$$

follows. Now the relation (8) can be written as (3) with (4).

COROLLARY 2.1. We have the relation

$$
\bigcup_{f \in G}\left\{w \mid w=\frac{1-z}{f(z)}, z \in D\right\}=\{w|| w-1 \mid<1\} .
$$

ProOF. The relation (9) follows from (3) for $|z| \rightarrow 1$.

COROLlaRY 2.2. For $z \in D$ and $f(z) \in G$, we have the sharp estimates

$$
1 /(1+|z|) \leqq|f(z) /(1-z)| \leqq 1 /(1-|z|)
$$

and

$$
|\arg (f(z) /(1-z))| \leqq \arcsin |z|,
$$

where for $z \neq 0$ equality holds only for the functions (4) at the "critical points"

$$
z= \pm|z| e^{-i \omega}
$$

and

$$
z=|z| e^{ \pm i(\pi / 2 \mp \omega-\arcsin |z|)}
$$

respectively.

PrOOF. The inequalities (10) with (12) and (11) with (13) follow from (3) on the basis of the inequalities $1-|z| \leqq|w| \leqq 1+|z|$ and $|\arg w| \leqq \arcsin |z|$, respectively. 
COROLlaRY 2.3. For each function

$$
f(z)=1+d_{1} z+d_{2} z^{2}+\cdots+d_{n} z^{n}+\cdots
$$

of the class $G$ in $D$, the inequalities

$$
\left|1+d_{1}+d_{2}+\cdots+d_{n}\right| \leqq 1, \quad n=1,2, \ldots,
$$

and

$$
\left|d_{n}\right| \leqq 2, \quad n=1,2, \ldots,
$$

hold with equality in (15) only for the functions (4) with $\omega \in[-\pi, \pi]$ and in (16) only for the function (4) with $\omega= \pm \pi$, i.e., $f(z)=(1-z) /(1+z)$.

ProOF. We write $w=f(z) /(1-z)$. Then (9) yields $|1 / w-1|<1$, i.e., $\operatorname{Re} w>\frac{1}{2}$. If

$$
w=\sum_{n=0}^{\infty} S_{n} z^{n}, \quad S_{n}=d_{0}+d_{1}+\cdots+d_{n}, d_{0}=1,
$$

the Borel-Carathéodory inequalities applied to $2 w-1$ yield $2\left|S_{n}\right| \leqq 2$, as required.

REMARK. The results in this paper can also be obtained by other methods and results due to Robertson $[1$, p. 331 , Theorem 1 ; 6, p. 318, Theorem 7; 7, pp. 385-386], Schild [8] and Pinchuk [9, pp. 722, 727-728, 732].

\section{REFERENCES}

1. M. S. Robertson, Univalent functions starlike with respect to a boundary point, J. Math. Anal. Appl. 81 (1981), 327-345.

2. A. Lyzzaik, On a conjecture of M. S. Robertson, Proc. Amer. Math. Soc. 91 (1984), 108110.

3. C. Carathéodory, Über den Variabilitätsbereich der Fourierschen Konstanten von positiven harmonischen Funktionen, Rend. Circ. Mat. Palermo 32 (1911), 193-217.

4. G. M. Goluzin, Geometrixeskaya teoriya funkcii kompleksnogo peremennogo, Izdanie vtoroe, Izdat. "Nauka", Moscow, 1966.

5. I. Ya. Asnevic and G. V. Ulina, Ob oblastyah znacenǐ̌ analiticeskih funckcǐ̌, predstavimyh integralom Stil' tessa, Vestnik Leningrad. Univ. 10 (1955), 31-42.

6. M. S. Robertson, Quasi-subordinate functions, Mathematical Essays Dedicated to A. J. Macintyre, Ohio Univ. Press, Athens, Ohio, 1970, pp. 311-330.

7., On the theory of univalent functions, Ann. of Math. 37 (1936), 374-408.

8. A. Schild, On starlike functions of order $\alpha$, Amer. J. Math. 87 (1965), 65-70.

9. B. Pinchuk, On starlike and convex functions of order $\alpha$, Duke Math. J. 35 (1968), 721734.

Department of Mathematics, Paissi Hilendarski University, 4000 Plovdiv, BULGARIA 